\title{
Cost-effectiveness of tenofovir gel in urban South Africa: model projections of HIV impact and threshold product prices
}

Fern Terris-Prestholt ${ }^{1 *+}$, Anna M Foss ${ }^{1+}$, Andrew P Cox ${ }^{1}$, Lori Heise ${ }^{1}$, Gesine Meyer-Rath ${ }^{1,2,3}$,

Sinead Delany-Moretlwe ${ }^{4}$, Thomas Mertenskoetter ${ }^{5}$, Helen Rees ${ }^{4}$, Peter Vickerman ${ }^{1}$ and Charlotte H Watts ${ }^{1}$

\begin{abstract}
Background: There is urgent need for effective HIV prevention methods that women can initiate. The CAPRISA 004 trial showed that a tenofovir-based vaginal microbicide had significant impact on HIV incidence among women. This study uses the trial findings to estimate the population-level impact of the gel on HIV and HSV-2 transmission, and price thresholds at which widespread product introduction would be as cost-effective as male circumcision in urban South Africa.
\end{abstract}

Methods: The estimated 'per sex-act' HIV and HSV-2 efficacies were imputed from CAPRISA 004. A dynamic HIV/STI transmission model, parameterised and fitted to Gauteng (HIV prevalence of $16.9 \%$ in 2008), South Africa, was used to estimate the impact of gel use over 15 years. Uptake was assumed to increase linearly to $30 \%$ over 10 years, with gel use in $72 \%$ of sex-acts. Full economic programme and averted HIV treatment costs were modelled. Cost per DALY averted is estimated and a microbicide price that equalises its cost-effectiveness to that of male circumcision is estimated.

Results: Using plausible assumptions about product introduction, we predict that tenofovir gel use could lead to a $12.5 \%$ and $4.9 \%$ reduction in HIV and HSV-2 incidence respectively, by year 15. Microbicide introduction is predicted to be highly cost-effective (under $\$ 300$ per DALY averted), though the dose price would need to be just $\$ 0.12$ to be equally cost-effective as male circumcision. A single dose or highly effective (83\% HIV efficacy per sex-act) regimen would allow for more realistic threshold prices ( $\$ 0.25$ and $\$ 0.33$ per dose, respectively).

Conclusions: These findings show that an effective coitally-dependent microbicide could reduce HIV incidence by $12.5 \%$ in this setting, if current condom use is maintained. For microbicides to be in the range of the most cost-effective HIV prevention interventions, product costs will need to decrease substantially.

Keywords: HIV, Modelling, Cost-effectiveness, Microbicides, South Africa, Introduction of new technologies, Economic analysis, Tenofovir, ARV-based prevention, Pre-exposure prophylaxis (PreP)

\section{Background}

There is an urgent need for effective HIV prevention methods that can be initiated by women. After a decade of little progress in HIV prevention interventions, recent trials have shown that antiretrovirals can be used to prevent HIV incidence and infectivity [1-4]. In 2010, the CAPRISA 004 trial in Kwazulu Natal, South Africa,

\footnotetext{
* Correspondence: Fern.Terris-Prestholt@lshtm.ac.uk

${ }^{\dagger}$ Equal contributors

${ }^{1}$ London School of Hygiene \& Tropical Medicine, London, UK

Full list of author information is available at the end of the article
}

showed that the incidence of HIV infection among women randomised to using a topical gel containing $1 \%$ of the antiretroviral tenofovir before and after sex, was reduced by $39 \%$ (95\% CI 6-60\%). In addition, women's risk of acquiring herpes simplex virus type-2 (HSV-2) was reduced by $51 \%(95 \%$ CI $22-70 \%)[5,6]$. This finding was groundbreaking, being the first clinical trial to show a statistically significant impact of a microbicide product on HIV incidence, and providing a proof of concept that a topically applied antiretroviral microbicide can reduce women's risk of HIV acquisition [7]. The FACTS 001 trial is now

\section{() Biomed Central}

(c) 2014 Terris-Prestholt et al.; licensee BioMed Central Ltd. This is an open access article distributed under the terms of the Creative Commons Attribution License (http://creativecommons.org/licenses/by/2.0), which permits unrestricted use, distribution, and reproduction in any medium, provided the original work is properly cited. 
seeking to confirm the CAPRISA results, and assess the generalizability of the findings across different settings [8]. Plans are underway to prepare for the possible introduction and manufacture of tenofovir gel. Once this trial is completed, its data will be combined with the CAPRISA 004 results and presented together for regulatory approval. This study aims to inform this process and the field of HIV more broadly.

Evidence from FACTS 001 and this cost-effectiveness analysis will be evaluated in the context of VOICE trial results that showed that daily administration of the same product and oral tenofovir did not achieve a significant reduction in HIV incidence due to adherence issues [9]. It appears that compliance to a daily regimen of gels or pills is not a viable option for African women in particular for younger women. However, given the positive findings from CAPRISA, there is still hope that a coitally-specific product may provide a feasible strategy for women who are unable to protect themselves with the current range of options available.

In parallel to clinical research, it is important to explore the potential population-level impact that tenofovir gel introduction could have on HIV incidence in different epidemic settings, and identify key factors that may influence the impact and cost-effectiveness of future product introduction. Towards this end, this paper uses modelling and the CAPRISA trial results to estimate the gel's likely level of protection against HIV and HSV-2 during each sex-act (here termed the 'imputed efficacy'), and the potential population-level impact on HIV and HSV-2 of gradual product introduction in an urban setting with high HIV prevalence and incidence in South Africa. Using South Africa specific unit cost data, the full cost of introducing microbicides into the public health sector is modelled first. Secondly, threshold product prices are identified at which point the costs per HIV infection averted are equal to that of voluntary medical male circumcision (VMMC) ( $\sim 1000$ per infection averted) [10], an intervention widely accepted as cost-effective and efficient. A range of scenarios are used to explore the effects of various assumptions on this threshold.

\section{Methods Setting}

For the modelling analysis, we considered product introduction in Gauteng Province, a highly urbanised province of South Africa with a population of reproductive age (aged 15 - 45 years) of 5.7 million. HIV prevalence among those aged $15-49$ was $16.9 \%$ in 2008 [11], with a higher prevalence among females (21.1\%) than males (11.2\%) documented in 2005 [12]. Commercial sex work is an important driver of the HIV epidemic in Gauteng [13], with behavioural surveys suggesting that $6.4 \%$ of female family-planning clinic attendees had transactional sex in the last four weeks [14].

\section{Microbicide introduction scenarios modelled}

A review of evidence and discussions with key stakeholders in South Africa and internationally were used to inform the microbicide introduction strategies considered in this paper [15]. As described in more detail below, we considered the provision of a microbicide through the public health system, assuming that mass media campaigns are used to increase awareness of the product availability; health providers are trained to provide the product, and they then routinely provide HIV testing, and counsel HIV negative clients about product use and the importance of adherence and repeated HIV testing [15].

As in the CAPRISA 004 trial, we consider a coitallydependent gel, with two doses used per sex-act. We assumed that the mean consistency of gel use is the same as in the CAPRISA 004 trial (two doses used in $72 \%$ of sex-acts) $)^{\mathrm{a}}$, irrespective of the type of partnership. In addition, a more pessimistic scenario of $50 \%$ gel use was considered.

In the CAPRISA trial, reported condom use increased from $79 \%$ in the first six months to $84 \%$ in the last six months $(\mathrm{P}<0.001)[5] .{ }^{\mathrm{b}}$ In this analysis we considered two potential scenarios. Firstly, we assumed that condom use is unaffected by gel use and HIV testing (and remains at the levels detailed in Additional file 1: Table S2 of the appendix). Secondly, we consider more pessimistic assumptions that there is a $5 \%$ or $10 \%$ absolute reduction in condom use among microbicide users.

In this analysis, we consider the impact on HIV and HSV-2 incidence of population-level gel distribution over 15 years, starting in January 2013. We assume that, following regulatory approval, tenofovir is made available to HIV-negative sexually active women attending public health facilities, alongside regular HIV testing.

Based on a review of the evidence on the rate of uptake of condoms and other new health technologies [16], we assume that for the first 10 years the proportion of HIVnegative women using the gel increases linearly, plateauing at $30 \%$, half the rate at which urban sexually active women are currently accessing modern contraceptives in Gauteng [17], for the remaining five years. A more optimistic scenario of $60 \%$ uptake after 10 years was also explored.

\section{Imputed efficacy calculations}

The CAPRISA 004 trial results represent an estimate of the average protection achieved across all users in the active arm of the trial, including those who used the gel consistently or intermittently, and those who did not use it at all [18]. As a result, for the modelling we need to impute the level of protection provided by the product against HIV and HSV-2 in each sex-act between 
a susceptible woman and an infected partner (that is, how much it reduces the probability of transmission during each act of intercourse). This was estimated by dividing the trial effectiveness by the percentage of all reported sex-acts in which gel was used $[5,6,18,19]$. Details of this calculation and the assumptions made are provided in the online Additional file 1. The imputed efficacy estimates were then used in the dynamic impact model. We also investigated the contribution of the gel's HSV-2 efficacy on HIV transmission and vice versa.

\section{Description of the model structure}

The HIV and HSV-2 impact projections were obtained using a population-level deterministic compartmental HIV/STI transmission model, adapted to enable the impact of the gradual introduction of a microbicide to be explored [20]. The mathematics underlying the dynamic transmission model are described in Vickerman et al. [21], and in the online Additional file. In brief, the model divides the heterosexual population into subgroups, with stratifications by sex, level of sexual activity and HIV/ STI infection status. Using data on the probabilities of disease transmission and progression from the epidemiological literature, established mathematical techniques are used to estimate how HIV spreads between the different subgroups over time [22,23]. The transmission of HSV-2, syphilis and gonorrhoea/chlamydia are simulated, including the facilitating effect of these STIs on HIV transmission, and the effect of HIV on increasing the rate of HSV-2 symptomatic recurrences and HSV-2 infectivity (during both asymptomatic and symptomatic stages of infection) [24]. The model also incorporates the higher HIV infectivity associated with primary and late-stage HIV infection (in the absence of HIV treatment), and the effect of ongoing STI treatment and antiretroviral therapy (ART) in the population on levels of HIV infectivity [25], of which more detail can be found in the online supplement.

\section{Fitting the model to the setting}

The model was parameterised and fitted using behavioural and epidemiological data from Gauteng, and complementary data from South Africa (Figures 1a, b, c and online Additional file 1). The approach used incorporates the uncertainty inherent in key model parameters by running the model over a large number (almost 4 billion) of permutations of model inputs. These are obtained by randomly sampling from the uncertainty ranges for each model input parameter (Additional file 1: Tables S2-S4) [21]. As is standard in HIV modelling, the model is seeded with a few HIV infected individuals $(0.5 \%$ in the general population and 5\% among female sex workers) in 1980 and run until 2028 (to model 15 years from 2013). A 'fit' is defined as a model simulation that lies within the $95 \%$ confidence intervals (CIs) of setting-specific HIV and STI prevalence data. The model was fitted to HIV data from three national surveys, using disaggregated male and female prevalence values when available (for 2002, 2005) and a combined prevalence for 2008 (five HIV prevalence data points in total for the general population) [11,12,26]; and also to HIV prevalence data among female sex workers (FSWs) (1997) and their clients (2000). Additionally, the model was fitted to syphilis and gonorrhoea/chlamydia prevalence data from FSWs (1997) [13,27]. HSV-2 data from the CAPRISA trial [5], adjusting for HIV status [11], was used as a proxy for the general population HSV-2 prevalence. Each combination of input values that fit to the observed prevalence data is used in the final impact projections, with the best-fit identified through least chi-squared error.

\section{Methods used for the economic evaluation of gel introduction}

Full programme costs are offset by the HIV care and treatment costs saved resulting from averting HIV infections to estimate incremental costs. We present the incremental cost-effectiveness ratios (ICER) in terms of dollar per disability adjusted life year (DALY) averted. Interventions with ICERs of less than per capita GDP (\$7160 for South Africa) per averted DALY are considered highly cost-effective and worth introducing [28], but require either additional resources or re-allocation of resources. We also identify the threshold microbicide prices where the cost per HIV infection averted is equal to the cost per HIV infection averted of VMMC in South Africa to better understand under which conditions microbicide introduction would be as cost-effective as VMMC.

The economic costs of a comprehensive microbicide distribution programme were estimated from the provider's perspective using an ingredients-based approach and unit cost from the South African public sector. This includes HIV testing [29], facility based provider training [30] and health facility costs, including staff, for the initiation visit and subsequent adherence counselling and gel distribution visits [31-33], 10\% product wastage [34], and periodic mass media campaigns [35]. This analysis assumes general population HIV testing is in place and the initial screening costs are not included.

All costs are presented in 2012 US dollars (\$). All future costs and effects are discounted to present values, using a $3 \%$ discount rate. The current price of a single dose of tenofovir gel as produced for the FACTS 001 trial is $\$ 1.60$ [36]. The predicted price of a packaged and labelled gel at low production scale (10 million doses) is $\$ 0.56$, while this could be as low as $\$ 0.17$ if distributed at large scale with a tube of gel and fillable paper applicators [36]. 

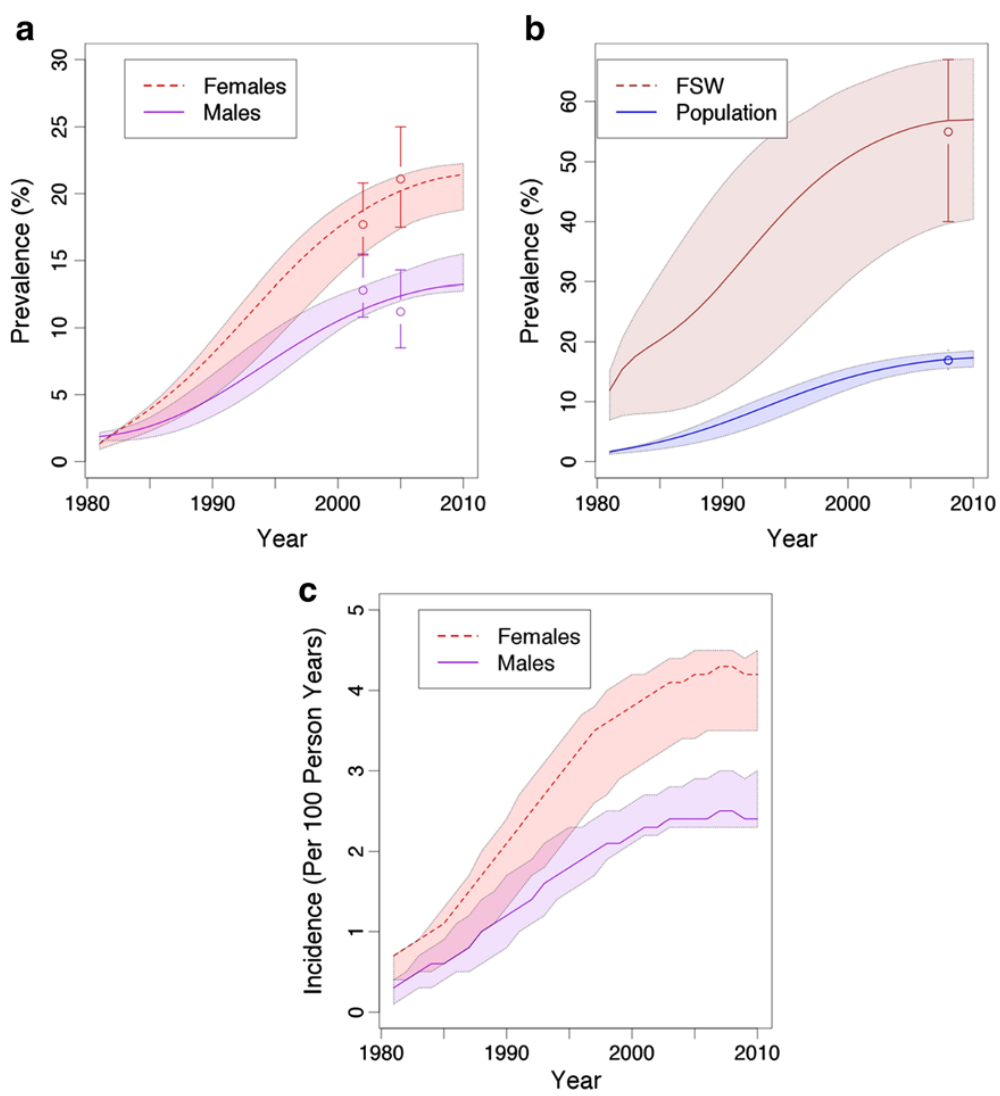

Figure 1 Model-projected HIV prevalence and incidence trends over time and data used to fit model*. a: HIV prevalence trends among general population males and females in 2002 and 2005. b: HIV prevalence trends among the general population and among female sex workers in 2008. c: HIV incidence trends among general population males and females. *The points with error bars for males and females in 2002 and 2005 represent the mean and 95\% confidence intervals derived from data [12,26]. The dotted line represents the model-projected female HIV prevalence while the solid line represents the model-projected male HIV prevalence. The shaded areas represent the range of prevalence estimates spanned by all model fits.

For the $52 \%$ of those in need of ART who are receiving ART [37], 2012 annual treatment costs are used (\$562 for first line ART and $\$ 1094$ for second line) [38], with an lifetime cost of $\$ 14,310$ assuming a near normal life expectancy due to ART [39]. For those without ART access, discounted lifetime HIV care costs of $\$ 3,080$ are included [33]. This gives a discounted weighted average HIV care and ART costs averted of $\$ 8,920$ per HIV infection averted. Though HSV-2 treatment with acyclovir is first line therapy for genital ulcer disease, these averted costs have not been included which will render the analysis on the conservative side.

An economic evaluation in terms of cost per DALY averted is estimated at a range of product prices with the central price being $\$ 0.25$ (including $\$ 1.60, \$ 0.56$, and \$0.17). Standard DALY estimates are used [40], with HIV disability weights drawn from the 2010 Global Burden of Disease study as presented in Ortblad [41]. Details of the economic evaluation methods are presented in the
Additional file 1: Table S5. To approximate the price thresholds where microbicide introduction is roughly as cost effective as VMMC scale up (\$1087 and \$1096 per infection averted to scale up to $60 \%$ and $80 \%$, respectively, from the current rate of $45 \%)$. To err on the conservative side, we use a $\$ 1,000$ per infection averted threshold. Threshold prices are estimated under a range of assumptions (number of annual HIV tests and clinic visits (two to six times per year for both) and dosing (one to two doses per sex-act), and epidemiological scenarios). A sensitivity analysis is undertaken to understand the possible impact on the threshold price of: the prices of ART $(-25 \%,-50 \%)$ and all other inputs $(+/-25 \%)$, discount rate $\left(0 \%, 6 \%^{\mathrm{c}}\right)$, and treatment access rates $(80 \%)$.

\section{Results}

Imputed efficacy and HIV incidence projections

The imputed efficacy calculations suggest that tenofovir gel reduced the per sex-act probability of HIV 
acquisition by $54 \%(95 \% \mathrm{CrI} 8-83 \%)$ and the per sex-act probability of HSV-2 acquisition by $71 \%$ (95\% CrI 3097\%). Using these as inputs in the population-level modelling, the projected impact on HIV incidence is shown in Figure 2.

Table 1 presents the projected impact of microbicide introduction under a number of scenarios. In the main intervention scenario with mean efficacies (30\% uptake achieved over 10 years, gel used in $72 \%$ of sex-acts and no reduction in condom use), we predict that the gel could lead to a $12.5 \%(95 \% \mathrm{CrI} 11.5-12.7 \%)$ relative reduction in HIV incidence and a 4.9\% (95\%CrI 4.7\%-5.5\%) relative reduction in HSV-2 incidence by year 15 . This is a reduction in HIV incidence in the whole population from 0.56 (95\%CrI 0.48-0.72) per 100 person-years to 0.49 (95\% CrI 0.42-0.64) per 100 person-years, and a reduction in HSV-2 incidence from 7.4 (95\%CrI 6.4-7.5) per 100 person-years to $7.1(95 \% \mathrm{Crl}$ 6.0-7.1) per 100 person years.

These reductions in incidence translate into averting 970 (95\%CrI 864-1,019) HIV infections and 1,471 (95\% CrI 1,336-1,543) HSV-2 infections per 100,000 population (Table 1) over 15 years. In this main scenario, one HIV infection and one HSV-2 infection is averted for every 1,317 (95\%CrI 1,266-1,481) and 868 (95\%CrI 831-977) microbicide protected sex-acts, respectively.

The impact projections are dependent upon the assumptions made regarding gel's efficacy, consistency and uptake, and the degree to which condom use may or may not be affected by gel introduction. For example, if the gel is used in $50 \%$ rather than $72 \%$ of sex-acts, then the number of HIV infections averted is reduced by $30 \%$ in relative terms.

Figure 2 shows how the HIV impact is affected by a number of modelling assumptions; the dark bars represent the main intervention scenario. Gel efficacy is very important, with a very highly efficacious gel potentially reducing HIV incidence by $19.0 \%$, while a gel with poor efficacy provides little population protection. Higher levels of uptake can have an important impact with a doubling of uptake resulting in a doubling of impact. There is also a $25 \%$ relative reduction in impact if condoms are used in 5\% fewer sex-acts but uptake and gel use is maintained (Figure 2).

Interestingly the model projections suggest the HSV-2 efficacy of the gel contributes little to HIV-impact. Even without any HSV-efficacy, the relative reduction in HIV incidence is still projected to be $12.0 \%$ after 15 years for the main intervention scenario compared to $12.5 \%$ if the gel is $71 \% \mathrm{HSV}-2$-efficacious. However, the HIV efficacy has an important influence on HSV-2 incidence: the relative reduction in $\mathrm{HSV}-2$ incidence is projected as $4.9 \%$ with $54 \% \mathrm{HIV}$ efficacy versus $3.5 \%$ if the gel has no HIV efficacy.

\section{Microbicide cost effectiveness and threshold prices}

Table 2 presents the cost-effectiveness of microbicide introduction. For the main intervention scenario (30\% uptake, $72 \%$ gel use, $54 \%$ HIV efficacy) with two gels per sex-act, three HIV tests and collection visits per year (after the initiation visit), the cost per DALY averted is $\$ 297$ at the price of $\$ 0.25$ per dose. At current trial

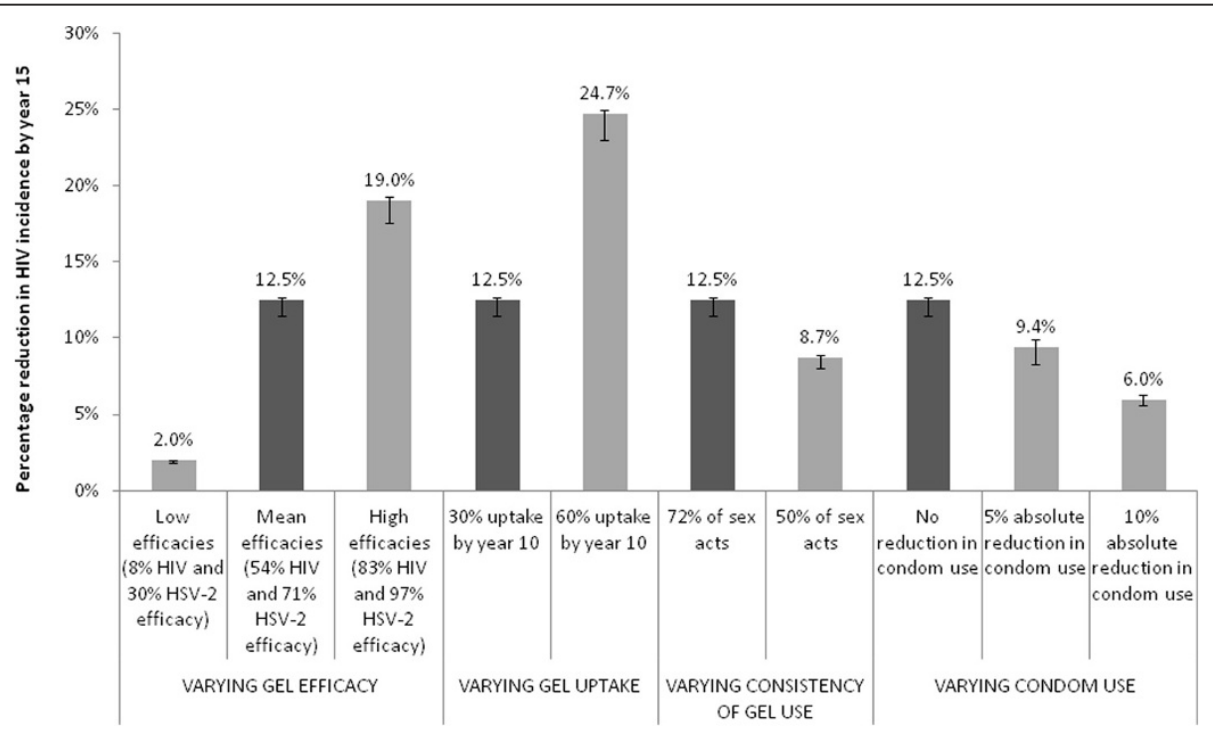

Figure 2 Projected population-level HIV impact in Gauteng for different assumptions about gel efficacy, uptake and use, and its influence on condom use ${ }^{* *}$. ${ }^{* *}$ Main shaded bars show best-fit model projections and error bars indicate range spanned by $95 \%$ of all model fits ( $2.5 \%$ to $97.5 \%$ percentile range or $95 \%$ credibility interval), with the dark bars illustrating the main scenario (54\% HIV efficacy and $71 \%$ HSV-2 efficacy, gel uptake reaching $30 \%$ by year 10 , gel used in $72 \%$ of sex-acts, and no reduction in condom use). 
Table 1 Projected impact from microbicide intervention*

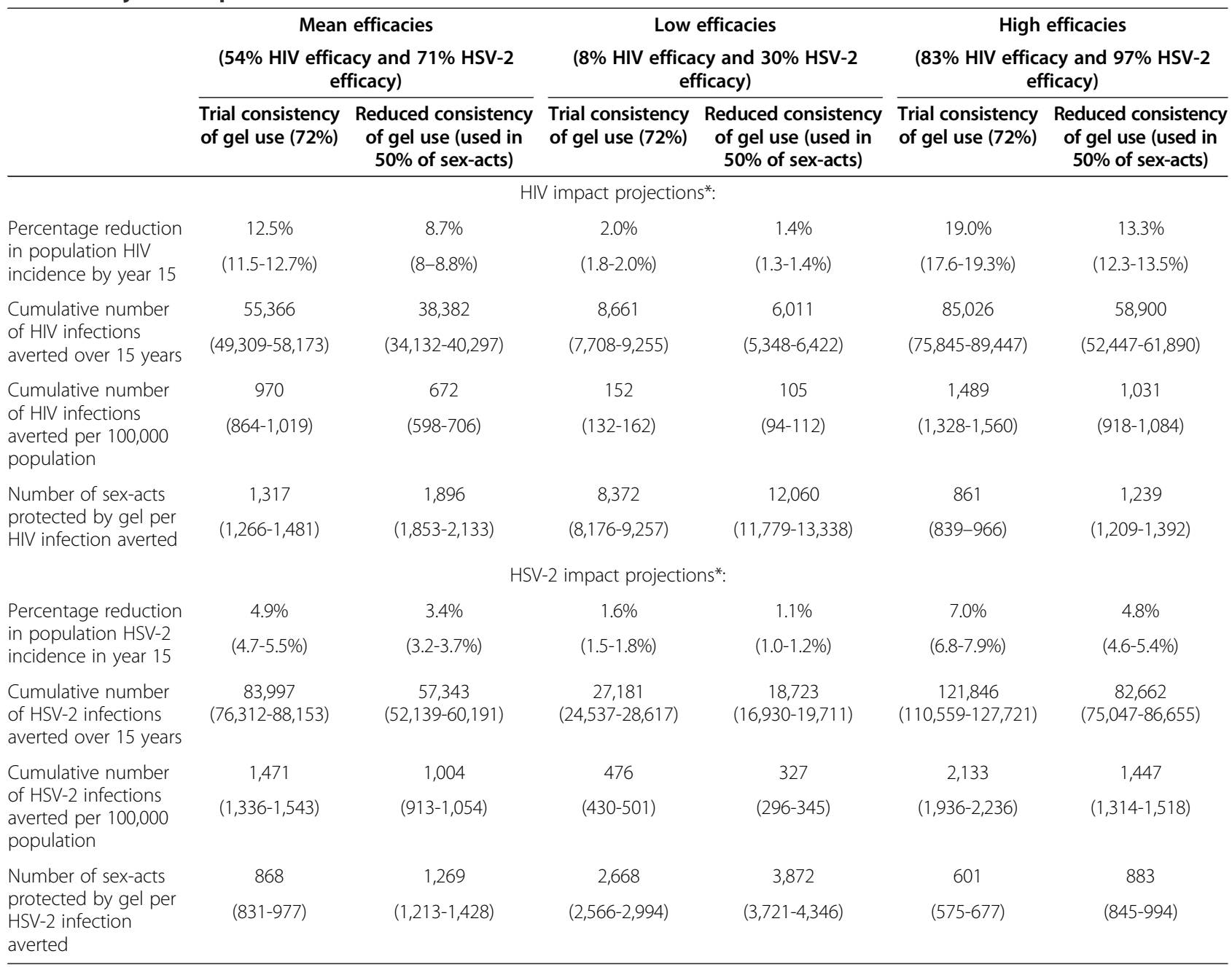

*Assuming gel only used by HIV-negative women, and levels of condom use are maintained after microbicide introduction.

Table 2 Cost-effectiveness and threshold prices under varying programmatic assumptions (2012 US\$)*

\begin{tabular}{|c|c|c|c|c|c|c|c|}
\hline & & \multicolumn{4}{|c|}{$\begin{array}{l}\text { Cost per DALY averted at } \\
\$ 0.25 \text { per dose }\end{array}$} & \multirow{2}{*}{\multicolumn{2}{|c|}{$\begin{array}{l}\text { Microbicide threshold prices } \\
\text { where \$/infection averted } \\
(\mathrm{IA})=\text { VMMC }(\sim \$ 1000 / \mathrm{IA}) \\
2 \text { gels per sex-act }\end{array}$}} \\
\hline & & \multicolumn{2}{|c|}{2 doses per sex-act } & \multicolumn{2}{|c|}{1 dose per sex-act } & & \\
\hline Baseline programme: 3 annual HIV tests, 3 gel collection visits & & $\$ 297$ & & & & $\$ 0.12$ & \\
\hline \multicolumn{8}{|l|}{ Variations to distribution services } \\
\hline \multirow[t]{3}{*}{ HIV tests per year } & 2 & $\$ 190$ & $-36 \%$ & $-\$ 29$ & $-137 \%$ & $\$ 0.19$ & $49 \%$ \\
\hline & 4 & $\$ 405$ & $36 \%$ & $\$ 186$ & $137 \%$ & $\$ 0.06$ & $-49 \%$ \\
\hline & 6 & $\$ 619$ & $108 \%$ & $\$ 401$ & $411 \%$ & Cannot be achieved & \\
\hline \multirow[t]{2}{*}{ Adherence counselling and gel collection visits } & 2 & $\$ 272$ & $-9 \%$ & $\$ 53$ & $-32 \%$ & $\$ 0.14$ & $12 \%$ \\
\hline & 6 & $\$ 374$ & $26 \%$ & $\$ 155$ & $97 \%$ & $\$ 0.08$ & $-35 \%$ \\
\hline Integrated adherence counselling and gel collection into HIV testing (3) & & $\$ 221$ & $-26 \%$ & $\$ 2$ & $-97 \%$ & $\$ 0.17$ & $35 \%$ \\
\hline
\end{tabular}


product costs of $\$ 1.60$, the cost per DALY averted would be $\$ 2,660$. At the lowest production cost, $\$ 0.17$ using cardboard applicators and large scale production, the cost per DALY averted would be as low as $\$ 157$. This variation emphasises the large contribution of the product in the total programme costs, ranging from $42 \%$ at the lowest price ( $\$ 0.17$ ) up to $87 \%$ at the highest price (\$1.60). At $\$ 0.25$, the product accounted for $51 \%$ of total programme costs, while HIV counselling and testing accounted for $38 \%$ of costs: each extra HIV test would increase the cost-effectiveness ratio by $36 \%$. The health facility costs of visits for adherence counselling and gel collection are relatively low, contributing only 9\% to programme costs: changes in collection frequency provide little savings or extra costs. However, a more integrated approach, with adherence counselling and gel distribution during the HIV testing visit, may be more attractive to clients and is more efficient, at \$221 per DALY averted. For microbicide introduction to be equally cost-effective as VMMC, product costs would need to drop below our lowest estimated production cost, to $\$ 0.12$ for the base scenario. By integrating gel collection and HIV testing, or by reducing the HIV tests to two per year, the cost threshold would just reach or surpass our lowest projected product cost.

We also model the cost-effectiveness and threshold prices for variations to gel uptake, use consistency, and
HIV efficacy, linked with the different impact scenarios (Table 3). A five percentage point absolute decrease in the percentage of sex-acts in which a condom is used would increase the cost to $\$ 548$ per DALY averted (i.e. worsen the cost-effectiveness), and a reduction in condom use of 10 percentage points would generate a cost of $\$ 1,219$ per DALY averted. A more efficacious gel could cost as much as $\$ 0.33$ per dose to be equally costeffective as VMMC, and at $\$ 0.25$ would generate an cost saving, indicated by the negative cost per DALY averted $(-\$ 14)$. Consistency of gel use (adherence) is also important, where only using gel in half the sex-acts reduces effectiveness by more than the reduction in costs, with cost of $\$ 468$ per DALY averted. This worsening is attributable to maintaining the annual fixed costs per facility and woman, while reducing gel use, and emphasises how important user behaviour will be in the products' ultimate cost-effectiveness. Uptake, however, has a relatively small impact on the break-even point: doubling uptake just increases the threshold price by $5 \%$, with both the fixed cost of reaching women and their gel use being reduced.

A sensitivity analysis of key cost assumptions is presented in the bottom half of Table 3 .

If a single dose regimen were found, the price could be as high as $\$ 0.25$, in line with expectations of production costs at scale. Though cost-effectiveness analyses have

Table 3 Cost-effectiveness and threshold gel prices under variations to introduction scenario assumptions and sensitivity analysis [2012 USD]

\begin{tabular}{|c|c|c|c|c|c|c|c|c|}
\hline \multicolumn{3}{|c|}{ Variations to introduction scenario } & \multirow[b]{2}{*}{$\begin{array}{l}\text { Consistency } \\
\text { of gel use }\end{array}$} & \multirow[b]{2}{*}{ HIV efficacy } & \multirow[b]{2}{*}{ \$/DALY averted } & \multirow[b]{2}{*}{$\%$ change } & \multirow[b]{2}{*}{ Threshold price } & \multirow[b]{2}{*}{$\%$ change } \\
\hline & $\begin{array}{l}\text { Reduction in } \\
\text { condom use }\end{array}$ & $\overline{\text { Coverage }}$ & & & & & & \\
\hline \multirow[t]{6}{*}{ Baseline } & $0 \%$ & $30 \%$ & $72 \%$ & $54 \%$ & $\$ 297$ & & $\$ 0.12$ & \\
\hline & $5 \%$ & $30 \%$ & $72 \%$ & $54 \%$ & $\$ 586$ & $97 \%$ & $\$ 0.03$ & $-74 \%$ \\
\hline & $10 \%$ & $30 \%$ & $72 \%$ & $54 \%$ & $\$ 1,219$ & $310 \%$ & cannot be achieved & \\
\hline & $0 \%$ & $60 \%$ & $72 \%$ & $54 \%$ & $\$ 285$ & $-4 \%$ & $\$ 0.13$ & $5 \%$ \\
\hline & $0 \%$ & $60 \%$ & $50 \%$ & $54 \%$ & $\$ 468$ & $58 \%$ & $\$ 0.03$ & $-78 \%$ \\
\hline & $0 \%$ & $30 \%$ & $72 \%$ & $83 \%$ & $-\$ 14$ & $-105 \%$ & $\$ 0.33$ & $166 \%$ \\
\hline \multicolumn{9}{|c|}{ Cost sensitivity analysis } \\
\hline \multicolumn{3}{|c|}{1 dose per sex-act } & & & $\$ 78$ & $-74 \%$ & $\$ 0.25$ & $100 \%$ \\
\hline \multicolumn{3}{|c|}{ Discount rate $0 \%$} & & & $-\$ 243$ & $-182 \%$ & $\$ 0.43$ & $244 \%$ \\
\hline \multicolumn{3}{|c|}{ Discount rate $6 \%$} & & & $\$ 525$ & $77 \%$ & cannot be achieved & \\
\hline \multicolumn{3}{|c|}{ ART costs $10 \%$ lower } & & & $\$ 344$ & $16 \%$ & $\$ 0.10$ & $-21 \%$ \\
\hline \multicolumn{3}{|c|}{ ART costs $25 \%$ lower } & & & $\$ 413$ & $39 \%$ & $\$ 0.06$ & $-53 \%$ \\
\hline \multicolumn{3}{|c|}{ All other input costs $25 \%$ higher } & & & $\$ 402$ & $35 \%$ & $\$ 0.06$ & $-48 \%$ \\
\hline \multicolumn{3}{|c|}{ All other input costs $25 \%$ lower } & & & $\$ 193$ & $-35 \%$ & $\$ 0.18$ & $48 \%$ \\
\hline \multicolumn{3}{|c|}{ Input $+25 \%$, ART-10\% } & & & $\$ 448$ & $51 \%$ & $\$ 0.04$ & $-69 \%$ \\
\hline \multicolumn{3}{|c|}{ Input $+25 \%$, ART-25\% } & & & $\$ 518$ & $74 \%$ & $-\$ 0.00$ & $-100 \%$ \\
\hline \multicolumn{3}{|c|}{ ART coverage $80 \%$} & & & $\$ 163$ & $-45 \%$ & $\$ 0.24$ & $90 \%$ \\
\hline
\end{tabular}


traditionally assumed an international discount rate of 3\%, more recent analyses have started using local discount rates to account for local time preferences which then better represent local decision making. The South African official discount rate in 2012 was 5.5\%. A 6\% discount rate has a very large impact on the cost-effectiveness: $\$ 525$ per DALY averted at $6 \%$ versus cost saving when discounting is removed in the costs. As the cost of ART provision has decreased dramatically over the past decade, we also explore the trade-off between treatment and prevention for lower ART provision costs. If ART costs were to drop by either $10 \%$ or $25 \%$, the cost-effectiveness of providing microbicides would worsen, increasing to $\$ 413$ per DALY averted. To account for variations in programme costs we explored changes to all other input prices by $+/-25 \%$, which would change cost-effectiveness ratio by $-/+35 \%$, respectively. In the most conservative case, where programme costs were increased by $25 \%$ and ART costs decreased by $25 \%$, the cost DALY averted is estimated at $\$ 518$. If ART coverage were expanded from $52 \%$ to $80 \%$, in line with the 2016 national target [37], then the threshold price of microbicides could be $\$ 0.24$. Though under most circumstances the explored microbicides are unlikely to be as cost-effective as VMMC, they would however reach a complementary target group (women) to which VMMC can never provide direct protection.

\section{Discussion and conclusions}

We have used detailed mathematical and economic modelling to estimate the impact and break-even price of tenofovir gel as introduced and distributed in urban South Africa. Our findings suggest that, for the main scenario considered, gel introduction could be an important addition to existing HIV prevention efforts, leading to a $12.5 \%$ (95\% CrI $11.5-12.7 \%)$ relative reduction in HIV incidence and a $4.9 \%(95 \% \mathrm{CrI} 4.7-5.5 \%)$ relative reduction in HSV-2 incidence over 15 years. The impact on HSV-2 is less than the impact on HIV, despite the higher efficacy against HSV-2, due to higher risk of HSV-2-infection for women.

Our findings suggest that the introduction of an effective gel could be cost saving at reasonable gel prices, in particular as ART access is expanded, and highly cost-effective even at current gel prices.

Indeed, using plausible assumptions about the full costs of programme delivery, we predict that the introduction of an HIV efficacious gel would be highly cost-effective ( $\$ 297$ per DALY averted) at a price of $\$ 0.25$, which is within the cost range (\$0.17-\$0.27) at large scale production of 100 million applicators [36]. In our model this scale of distribution is achieved from year 9 onwards. If South Africa reaches its target of $80 \%$ on ART or a single dose regimen is found to be effective, we can expect microbicides to be around the same cost per HIV infection averted as VMMC.

Even at current FACTS 001 trial production prices (\$1.60) [36], the incremental cost per DALY averted would be around $\$ 2,660$, which is well below the commonly applied cost-effectiveness threshold of $1^{*}$ GDP $(\$ 7,610$ for South Africa) [42].

Using the trial estimates of $72 \%$ adherence, we impute that the HIV and HSV-2 per sex-act efficacies of tenofovir gel are 54\% (95\% CrI 8-83\%) and 71\% (95\%CrI 30-97\%), respectively. Although these estimates are based upon the trial measures of gel adherence reporting, we considered that these are reasonably reliable, as gel applicator returns were found to correlate well with vaginal tenofovir concentration measured in a pharmacokinetics sub-study [43]. We are also reassured about the validity of our imputed efficacy estimates because a secondary analyses of the effectiveness among 'high adherers' in the trial (i.e. women who used both doses of the gel in over $80 \%$ of sexacts) found that the risk of HIV acquisition in this population was reduced by 54\% (95\%CI 4-80\%) [5]. However, the wide bounds on the imputed efficacy estimates of tenofovir gel, due to the wide confidence intervals on the trial effectiveness estimates, lead to a wide range of population-level impact estimates predicted by the model.

This analysis builds upon our previous modelling of the potential impact of microbicide introduction in different settings [44-48], as well as the work by Williams et al. [48], Verguet et al. [39] and Walensky et al. [49-51]. Additional strengths here include that the dynamic model is carefully parameterised and rigorously fitted to a specific setting over multiple time points, including fitting to HIV and STI infection status by sex, as well as incorporating the HSV-2-efficacy of tenofovir found in CAPRISA 004 and the related bi-directional interaction between HSV-2 and HIV and the dynamic effects of gel on the HIV and HSV-2 epidemic. Walensky et al. [50] included only the first generation of HIV infections averted. Numerous programme implementation components were also included in our analysis, such as training, mass media and facility distribution costs, to provide a more comprehensive approach to costs than comparable studies to date. However, we did not account for potential toxicity or ART resistance as in Walensky et al. [50], because the concerns in this area are limited, as the authors themselves acknowledge. Moreover, the scenarios that we have modelled are less optimistic than those considered previously [49]. This study makes lower assumptions regarding the likely coverage that can be achieved, more optimistic assumptions about the impact of ART on life expectancy and the discounting of future DALYs averted (an sometime omitted step [51]. Consequently, we produce a far more conservative estimate of impact and cost-effectiveness. 
Our modelling stresses the importance of maintaining condom use following microbicide introduction, to avoid large reductions in impact and cost-effectiveness. This issue is particularly of concern if condom use is already high [48], and for a microbicide with lower efficacy, as concomitant reductions in condom use could even lead to increases in the overall number of infections [48]. Similarly, our analyses highlight the degree to which the costs of gel introduction will be affected by programme costs, including the unit cost of gels, and the required frequency of HIV testing. For this reason, further research is needed to identify the optimal spacing between HIV testing, with the aim of achieving an appropriate balance between what may be required to minimise the potential for resistance and be feasible for women and health systems. Similarly, research to explore whether a single dose of tenofovir-containing gel, applied prior to sex, could be effective at reducing a woman's risk of acquiring HIV is needed [52], to help reduce the product costs, as well as make gel use less burdensome for women.

The fact that $17 \%$ of VOICE participants reported engaging in anal intercourse during the 3-month period prior to enrolment highlights an important area for future modelling work [9]. For such modelling, data is needed from different sites and population groups on the frequency of anal sex over a specific timeframe (e.g. past 3 or 6 months), and whether a condom and/or gel is used during the last anal sex-act.

Although it is tempting to draw broader, national or regional conclusions from this analysis, our model projections may not be generalisable to other settings with different epidemic and behavioural profiles and different price levels. However, the impact we predict compares favourably with previous modelling of a hypothetical microbicide introduced into an area within Gauteng [19], and with modelling studies of the impact of male circumcision on HIV incidence in various settings of sub-Saharan Africa [53-55], matched to the same intervention assumptions made here [56].

The results are also highly sensitive to the discount rate used. Our central estimate used a 3\% discount rate as commonly applied in international studies. However, when using the upper bound of $6 \%$, just above the South African national discount rate of $5.5 \%$, the cost-effectiveness of microbicides would worsen dramatically by $77 \%$. Although not a realistic price to expect during mass-production, the programme's incremental cost-effectiveness, at the current product cost of $\$ 1.60$ per dose would still be highly cost-effective at $\$ 2,825$ per DALY averted even using a $6 \%$ discount rate.

Some have questioned the viability of tenofovir gel given recent disappointing evidence of low user adherence in the VOICE trial. That users have a difficult time with adherence, however, should come as no surprise.
Inconsistent adherence is the norm for both medications and any prevention methods that are user-controlled. Nonetheless, every indication is that the South African government and international donors are prepared to seek licensure for tenofovir gel should the results of the CAPRISA trial be sustained in the on-going FACTS 001 trial. Given that policymakers will soon face a key decision point regarding the possible introduction and scale up of tenofovir gel, the findings of this article are highly relevant. Despite intuitions to the contrary, it appears from the results of VOICE, FEMPREP, and other trials of daily oral or vaginal prophylaxis, that women seem better able to adhere to the coitally-dependent BAT-24 regimen than daily use.

Moreover, even if the attention of the wider field has shifted towards long-acting modalities such as the vaginal ring and injection, these products are still many years from licensure and there is undoubtedly benefit of beginning to prepare consumers for using ARVs to prevent infection. There will always be a market for methods like the vaginal gel that will likely not require on-going HIV testing and that can be positioned as a sexual lubricant with additional HIV fighting powers. Though highly cost-effective prevention options are available for men (VMMC and male condoms), there is still a great need to seek coitally-dependent options that women can initiate, such as microbicides, in whichever formulation.

This study has used epidemiological and cost modelling to estimate the impact of microbicide introduction among women of the general population in the Gauteng Province in South Africa. We used highly conservative assumptions to estimate gel impact and found that microbicides have the potential to significantly reduce HIV incidence among women in Gauteng. This is the most comprehensive model of the costs of microbicide introduction available in the literature to date and, even under the very conservative assumptions this analysis, estimated that microbicide introduction is very likely to be highly cost-effective. However this analysis also highlighted the importance of continued strong condom distribution and counselling programmes to maintain condom use and the need for identifying innovative approaches for supporting microbicide adherence.

\section{Endnotes}

${ }^{a}$ Adherence estimates based on applicator returns for the remaining 884 women indicate that, on average, $72.2 \%$ (median $=60.2 \%$ ) of self-reported sex-acts in the last 30 days were covered by two doses of gel.

${ }^{\mathrm{b}}$ It should be noted that condom use outside the trial setting is lower and our approach will therefore underestimate the impact of microbicides on HIV incidence.

${ }^{\mathrm{C}}$ The $6 \%$ discount rate is consistent with the South African central bank discount rate of 5.5\% [57]. 


\section{Additional file}

Additional file 1: Table S1. Average additional life expectancy and reduction in transmission probabilities for the population attributable to ART treatment. Table S2. Behavioural and demographic parameters used in the model for Gauteng Province. Table S3. Biological parameters used in the model for Gauteng Province. Table S4. Data used in model fitting: Prevalence of Sexually Transmitted Infections - Gauteng/South Africa. Table S5. Cost inputs, assumptions and sources.

\section{Abbreviations}

ART: Antiretroviral therapy; Cl: Confidence interval for data estimates; Crl: Credibility interval for model projections - defined as the 2.5 to $97.5 \%$ percentile range; DALY: Disability adjusted life year; DfID: Department for International Development; FSW: Female sex workers; HIV: Human immunodeficiency virus; HSV-2: Herpes simplex type 2; ICER: Incremental cost-effectiveness ratio; IPM: International Partnership for Microbicides; MDP: Microbicide development programme.

\section{Competing interests}

I have read the journal's policy and have the following conflicts: AF, FTP, AC, $\mathrm{PV}, \mathrm{CW}$ received a grant from IPM in the initial development of these models. At the time, TM was employed by IPM. IPM aims to promote the development and delivery of safe and effective microbicides around the globe. LH was previously the director for the Global Campaign for Microbicides.

\section{Authors' contributions}

Conceptualised the study: AF, PV, FTP, TM, AC, LH,CW; Developed the model: $P V$ and $A F$; Undertook the impact modelling: $A C$ and $A F$ and $P V_{;}$Undertook the cost modelling: FTP; Wrote the first draft of the manuscript: AF, FTP. Contributed to the writing of the manuscript: LH, AC, GMR, SDM, TM, HR, PV, CW; Criteria for authorship read and met: AF, FTP, AC, LH, GMR, SDM, TM, HR, $P V, C W$. Agree with manuscript results and conclusions AF, FTP, AC, LH, GMR, SDM, TM, HR, PV, CW. All authors read and approved the final manuscript.

\section{Acknowledgements}

The authors are grateful for the ongoing support, advice and input from Céline Mias, Saul Walker, Youssef Tawfik and Vimala Raghavendran. We thank David Friend for contributing likely tenofovir gel product costs under various production scales. The authors are extremely grateful to Christine Michaels, Chiweni Chimbwete, Emeka Okonji, Richard Hayes, Mitzy Gafos, Lilani Kumaranayake, Jocelyn Moyes, Mags Beksinska, Sibongile Walaza, Claire von Mollendorf, Jennifer Smit, Anna Vassall and Michelle Remme for their support and input into this work, and to Salim S. Abdool Karim and Brian Williams for sharing trial data on condom and gel use and. The authors are also indebted to all the participants of the various microbicide trials.

\section{Funding}

The Bill \& Melinda Gates Foundation provided grant support for some of the activities described in this paper. This research project was conducted in conjunction with the International Partnership for Microbicides (IPM), funded by the European Union, and contributes to the Microbicides Development Programme (MDP). MDP is a partnership of African and European academic/ government institutions and commercial organisations. MDP is funded by the British Government Department for International Development (DFID) and the UK Medical Research Council. AF, FTP, AC, PV, GMR and CW were also members of the DfID Research Programme Consortium for Research and Capacity Building in Sexual and Reproductive Health and HIV in Developing Countries of the LSHTM.

\section{Author details}

${ }^{1}$ London School of Hygiene \& Tropical Medicine, London, UK. ${ }^{2}$ Health Economics and Epidemiology Research Office, Department of Medicine, Faculty of Health Sciences, University of the Witwatersrand, Johannesburg, South Africa. ${ }^{3}$ Center for Global Health and Development, Boston University, Boston, USA. "Wits Reproductive Health and HIV Research Institute, Johannesburg, South Africa. ${ }^{5}$ International Partnership for Microbicides, Silver Spring, MD, USA.
Received: 21 March 2013 Accepted: 24 December 2013

Published: 9 January 2014

\section{References}

1. Cohen M, Chen Y, McCauley M, Gamble T, Hosseinipour M, Kumarasamy N, et al: Prevention of HIV-1 infection with early antiretroviral therapy. N Engl J Med 2011, 365:493-505.

2. Grant R, Lama J, Anderson P, McMahan V, Liu A, Vargas L, et al: Preexposure chemoprophylaxis for HIV prevention in men who have sex with men. N Engl J Med 2010, 363:2587-2599.

3. Baeten J, Donnell D, Ndase P, et al: Antiretroviral prophylaxis for HIV prevention in heterosexual men and women. N Engl J Med 2012, 367:399-410.

4. Thigpen MC, Kebaabetswe PM, Paxton LA, Smith DK, Rose CE, Segolodi TM, Henderson FL, Pathak SR, Soud FA, Chillag KL, et al: Antiretroviral Preexposure Prophylaxis for Heterosexual HIV Transmission in Botswana. N Engl J Med 2012, 367:423-434.

5. Karim QA, Karim SS, Frohlich JA, Grobler AC, Baxter C, Mansoor LE, Kharsany AB, Sibeko S, Mlisana KP, Omar Z, et al: Effectiveness and Safety of Tenofovir Gel, an Antiretroviral Microbicide, for the Prevention of HIV Infection in Women. Science 2010. Epub:http://www.sciencemag.org/ content/early/2010/07/19/science.1193748? explicitversion=true.

6. Karim SSA: Results of the CAPRISA 004 trial of tenofovir gel. In XVIII International AIDS Conference 18-23 July. Vienna, Austria; 2010.

7. Cates W Jr: After CAPRISA 004: time to re-evaluate the HIV lexicon. Lancet 2010, 376:495-496.

8. FACTS 001 Study. http://www.facts-consortium.co.za/?page_id=83.

9. Marrazzo J, Ramjee G, Nair G, Palanee T, Mkhize B, Nakabiito C, Taljaard M, Piper J, Gomez-Feliciano K, Chirenje M, VOICE Study Team: Pre-exposure Prophylaxis for HIV in Women: Daily Oral Tenofovir, Oral Tenofovir/Emtricitabine, or Vaginal Tenofovir Gel in the VOICE Study (MTN 003). In 20th Conference on Retroviruses and Opportunistic Infections; March 3-6. Atlanta, Georgia, USA; 2013.

10. Bärnighausen T, Bloom DE, Humair S: Economics of antiretroviral treatment vs. circumcision for HIV prevention. Proc Natl Acad Sci U S A 2012, 109:21271-21276.

11. Shisana O, Rehle T, Simbayi L, Zuma K, Jooste S, Pillay-van-Wyk V, Mbelle N, Van Zyl J, Parker W, Zungu N, et al: South African national HIV prevalence, incidence, behaviour and communication survey. A turning tide among teenagers?. Cape Town: HSRC Press; 2009.

12. Shisana O, Rehle T, Simbayi L, Parker W, Zuma K, Bhana A, Connolly C, Jooste S, Pillay V: South African National HIV Prevalence. Behaviour and Communication Survey: HIV Incidence; 2005

13. Dunkle K, Beksinka M, Rees H, Ballard R, Htun Y, Wilson ML: Risk factors for HIV infection among sex workers in Johannesburg, South Africa. Int J STD AIDS 2005, 16:256-261.

14. RHRU: Reproductive Health Research Unit Behavioural Sentinel Survey Data Report - Clients of family planning - Gauteng. Johannesburg, South Africa: Reproductive Health Research Unit, University of the Witwatersrand; 2000.

15. Watts C, Foss A, Kumaranayake L, Cox A, Terris-Prestholt F, Vickerman P: Identifying optimal strategies for microbicide distribution in India and South Africa: Modelling and cost-effectiveness analyses. In International Partnership for Microbicides with the London School of Hygiene and Tropical Medicine. Brussels and London; 2008.

16. Rees H: Service Delivery and Demand Challenges in Introducing new health products. Mexico City: Microbicide Access Forum; 2008.

17. Department of Health Medical Research Council, OrcMacro: South Africa Demographic and Health Survey 2003. Pretoria: Department of Health Republic of South Africa; 2007.

18. Heise L, Watts C, Foss A, Vickerman P, Trussell J, Hayes R, McCormack S: Apples and oranges? Interpreting success in HIV prevention trials. Contraception 2011, 83:10-15.

19. Vickerman P, Foss A, Watts C: Using modeling to explore the degree to which a microbicide's sexually transmitted infection efficacy may contribute to the HIV effectiveness measured in phase 3 microbicide trials. J Acquir Immune Defic Syndr 2008, 48:460-467.

20. Vickerman P, Watts C, Delany S, Alary M, Rees $H$, Heise L: The importance of context: model projections on how microbicide impact could be affected by the underlying epidemiologic and behavioral situation in 2 African settings. Sex Transm Dis 2006, 33:397-405.

21. Vickerman P, Foss AM, Pickles M, Deering K, Verma S, Demers E, Moses S, Alary M, Boily M-C: Is the Indian HIV epidemic driven by commercial sex? A modelling analysis from south India. AIDS 2010, 24:2563-2572. 
22. Garnett GP, Anderson RM: Strategies for limiting the spread of HIV in developing countries: conclusions based on studies of the transmission dynamics of the virus. J Acquir Immune Defic Syndr Hum Retrovirol 1995, 9:500-513.

23. Weinstein MC, Graham JD, Siegel JE, Fineberg HV: Cost-effectiveness analysis of AIDS prevention programs: concepts, complications and illustrations. In Confronting AIDS. Edited by Turner CF, Miller HG, Moses LE. Washington DC: National Academy Press; 1989:471-499.

24. Foss AM, Vickerman PT, Mayaud P, Weiss H, Ramesh BM, Reza-Paul S, Washington R, Blanchard J, Moses S, Lowndes CM, et al: Modelling the interactions between HSV-2 and HIV: implications for the HIV epidemic in southern India. Sex Transm Infect 2011, 87:22-27.

25. Wawer MJ, Gray RH, Sewankambo NK, Serwadda D, Li X, Laeyendecker O, Kiwanuka N, Kigozi G, Kiddugavu M, Lutalo T, et al: Rates of HIV-1 transmission per coital act, by stage of HIV-1 infection, in Rakai, Uganda. J Infect Dis 2005, 191:1403-1409.

26. Shisana O, Simbayi L: Nelson Mandela/HSRC study of HIV/AIDS. South African National HIV Prevalence, Behavioural Risks and Mass Media. The Human Sciences Research Council: Cape Town; 2002.

27. Johnson LF, Coetzee DJ, Dorrington RE: Sentinel surveillance of sexually transmitted infections in South Africa: a review. Sex Transm Infect 2005, 81:287-293.

28. CHOosing Interventions that are Cost Effective (WHO-CHOICE) [http://www.who.int/choice/costs/CER_thresholds/en/]

29. Bassett IV, Giddy J, Nkera J, Wang B, Losina E, Lu Z, Freedberg KA, Walensky RP: Routine voluntary HIV testing in Durban, South Africa: the experience from an outpatient department. J Acquir Immune Defic Syndr 2007, 46:181-186.

30. Terris-Prestholt F, Kumaranayake L, Obasi Al, Cleophas-Mazige B, Makokha M, Todd J, Ross DA, Hayes RJ: From Trial Intervention to Scale-Up: Costs of An Adolescent Sexual Health Program in Mwanza, Tanzania. Sex Transm Dis 2006, 33:S133-139.

31. Terris-Prestholt F, Michaels C, Kumaranayake L, Meyer-Rath G, Watts C: Costing Microbicide Distribution: Estimating cost-effectiveness \& modelling potential role out. Entebbe, Uganda: MDP301 Annual Investigators Meeting; 2009.

32. Meyer-Rath G: Public Sector Salary Costs. ; 2009. Personal Communicatuion.

33. Cleary S, Bouille A, McIntyre D, et al: Cost-effectiveness of Antiretroviral Treatment for HIV-positive Adults in a South African Township. Cape Town: University of Cape Town, Health Systems Trust, Medecins Sans Frontieres; 2004.

34. Gilson L, Mkanje R, Grosskurth H, Mosha F, Picard J, Gavyole A, Todd J, Mayaud P, Swai R, Fransen L, et al: Cost-effectiveness of improved treatment services for sexually transmitted diseases in preventing HIV-1 infection in Mwanza Region, Tanzania. Lancet 1997, 350:1805-1809.

35. Bollinger L, Stover J, Boulle A, Cleary S: Database of Unit Costs for HIV and AIDS interventions in South Africa: Resource Needs for HIV/AIDS: Model for estimating resources needed for prevention, care and mitigation. In Book Database of Unit Costs for HIV and AIDS interventions in South Africa: Resource Needs for HIV/AIDS: Model for estimating resources needed for prevention, care and mitigation. City: Centre for Economic Governance and AIDS in Africa; 2006.

36. Friend D: Tenofovir $1 \%$ gel, Current Prices and Expected Prices at Different Scales. 2012. Personal communication.

37. Johnson L: Access to antiretroviral treatment in South Africa, 2004-2011. S Afr J HIV Med 2012, march:22-27.

38. Meyer-Rath G: National ART Cost Model, South Africa. Johannesburg: Health Economics and Epidemiology Research Office, Boston University/University of the Witwatersrand; 2011

39. Johansson KA, Robberstad B, Norheim OF: Further Benefits by Early Start of HIV Treatment in Low Income Countries: Survival Estimates of Early Versus Deferred Antiretroviral Therapy. AIDS Res Ther 2010, 7:3. doi:10.1186/1742-6405-7-3.

40. Fox-Rushby JA, Hanson K: Calculating and presenting disability adjusted life years (DALYs) in cost-effectiveness analysis. Health Policy Plan 2001, 16:326-331.

41. Ortblad KF, Lozano R, Murray CJ: The burden of HIV: insights from the GBD 2010. AIDS (London, England) 2013.

42. GDP per capits (current US\$). http://data.worldbank.org/indicator/NY.GDP PCAP.CD.
43. Kashuba AD, Abdool Karim SS, Kraft E, White N, Sibeko S, Werner L, Mansoor LE, Gengiah T, Sidhoo S, Abdool Karim Q: Do systemic and genital tract tenofovir concentrations predict HIV seroconversion in the CAPRISA 004 tenofovir gel trial? In XVIII International AIDS Conference 18-23 July. Vienna, Austria; 2010.

44. Watts $\mathrm{CH}$, Kumaranayake L, Vickerman P, Terris-Prestholt F: The public health impact of microbicides: model projections. Report from the Public Health Benefits Working Group. In The Rockefeller Foundation Microbicide Initiative. 2002. http://www.global-campaign.org/clientfiles/rep7_publichealth.pdf.

45. Watts C, Vickerman P: The impact of microbicides on HIV and STD transmission: model projections. AIDS 2000, 15:S43-S44.

46. Vickerman P, Devine A, Foss AM, Delany-Moretlwe S, Mayaud P, Meyer-Rath G: The cost-effectiveness of herpes simplex virus-2 suppressive therapy with daily aciclovir for delaying HIV disease progression among HIV-1-infected women in South Africa. Sex Transm Dis 2011, 38:401-409.

47. Terris-Prestholt F, Kumaranayake L, MacPhail C, Rees H, Watts C: Determinants of South African Women's Demand for New Barrier Methods and their Distribution: Analysis of a Discrete Choice Experiment. In IAEN; 1-2 August; Cuernavaca; 2008.

48. Foss $\mathrm{AM}$, Vickerman PT, Heise $\mathrm{L}$, Watts $\mathrm{CH}$ : Shifts in condom use following microbicide introduction: should we be concerned? AIDS 2003, 17:1227-1237

49. Williams BG, Karim SSA, Karim QA, Gouws E: Epidemiological impact of tenofovir gel on the HIV epidemic in South Africa. J Acquir Immune Defic Syndr 2011, 58:207-210.

50. Walensky RP, Park JE, Wood R, Freedberg KA, Scott CA, Bekker LG, Losina E, Mayer KH, Seage GR 3rd, Paltiel AD: The cost-effectiveness of pre-exposure prophylaxis for HIV infection in South African women. Clin Infect Dis 2012, 54:1504-1513.

51. Verguet $S$, Walsh JA: Vaginal microbicides save money: a model of cost-effectiveness in South Africa and the USA. Sex Transm Infect 2010 $86: 212-216$

52. MDP Statement on the Discontinuation of Tenofovir gel in the VOICE Trial. http://www.mdp.mrc.ac.uk/

53. Gray RH, Li X, Kigozi G, Serwadda D, Nalugoda F, Watya S, Reynolds SJ, Wawer M: The impact of male circumcision on HIV incidence and cost per infection prevented: a stochastic simulation model from Rakai, Uganda. AIDS 2007, 21:845-850.

54. Hallett TB, Singh K, Smith JA, White RG, Abu-Raddad LJ, Garnett GP: Understanding the impact of male circumcision interventions on the spread of HIV in southern Africa. PLoS One 2008, 3:e2212.

55. White RG, Glynn JR, Orroth KK, Freeman EE, Bakker R, Weiss HA, Kumaranayake L, Habbema JD, Buve A, Hayes RJ: Male circumcision for HIV prevention in sub-Saharan Africa: who, what and when? AIDS 2008, 22:1841-1850

56. Cox AP, Foss AM, Vickerman PT, Chimbwete C, Okonji E, Terris-Prestholt F, Beksinska M, Walaza S, von Mollendorf C, Smit J, et al: Making realistic impact projections for new HIV prevention technologies: looking beyond a product's efficacy. In 18th International Society for Sexually Transmitted Diseases Research; 28 June - 1 July. London, UK: 2009.

57. Central Bank Rates. www.cbrates.com.

\section{doi:10.1186/1471-2334-14-14}

Cite this article as: Terris-Prestholt et al:: Cost-effectiveness of tenofovir gel in urban South Africa: model projections of HIV impact and threshold product prices. BMC Infectious Diseases 2014 14:14. 